\title{
Delta-6-desaturase gene polymorphism is associated with lipoprotein oxidation in vitro
}

\author{
Tiina Solakivi ${ }^{1}$, Tarja Kunnas ${ }^{1}$, Olli Jaakkola ${ }^{2}$, Jaana Renko ${ }^{1}$, Terho Lehtimäki ${ }^{3}$ and Seppo T Nikkari ${ }^{1,3^{*}}$
}

\begin{abstract}
Background: Oxidative modification of low-density lipoprotein (LDL) is a key event in the oxidation hypothesis of atherogenesis. We have previously shown that HDL does not protect LDL from oxidation in vitro, but is in fact oxidized fastest of all lipoproteins due to its rich polyunsaturated fatty acid (PUFA) composition, which is oxidation promoting. Evidence has accumulated to show that in addition to diet, common polymorphisms in the fatty acid desaturase (FADS) gene cluster have very marked effects on human PUFA status. There is a deletion [T/-] in the promoter region of the $\Delta^{6}$-desaturase gene (FADS2, rs 3834458), which has a direct inhibitory influence on production of PUFA from linoleic and alpha-linolenic acid. To investigate the possible role of rs 3834458 in lipoprotein modification, oxidation of $\mathrm{LDL}$ with $\mathrm{HDL}_{2}$ or $\mathrm{HDL}_{3}$ were analyzed from plasma of 58 free-living individuals.

Results: Total eicosapentaenoic acid and arachidonic acid were significantly decreased in plasma from the 10 subjects homozygous for the deletion in FADS2 rs 3834458. When the isolated LDL and HDL 2 were subjected to $\mathrm{Cu}^{2+}$-induced oxidation, these subjects showed decreased rate of appearance $(p=0.027)$ and the final concentration of conjugated dienes $(p=0.033)$ compared to the other genotypes. For oxidation of $L D L$ with $H D L_{3}$, the final concentration of conjugated dienes was also significantly decreased in subjects with $[-/-]$ compared with $[T / T]$ and $[T /-](p=0.034)$.

Conclusion: We conclude that FADS2 genotype may play a role in peroxidation susceptibility of lipoproteins.
\end{abstract}

\section{Background}

Dietary and endogenously produced fatty acids are known to modulate the metabolism of lipids and lipoproteins and therefore also to be involved in cardiovascular and metabolic diseases [1]. Polyunsaturated fatty acids (PUFA) are classified into two families, the $\mathrm{n}-6$ and $\mathrm{n}-3$ series.

Linoleic acid (18:2 n-6) and $\alpha$-linolenic acid (18:3 n-3) have become known as essential fatty acids (EFA) because they cannot be synthesized de novo by mammals, including humans, but are nevertheless necessary for proper physiological functioning [1]. The human body can then modify fatty acids by $\Delta 6$ and $\Delta 5$ desaturases and elongases to their respective metabolites along the pathways shown in Figure 1 to meet the metabolic needs. There is a common deletion $[\mathrm{T} / \mathrm{-}]$ in the promoter region of the $\Delta 6$

\footnotetext{
* Correspondence: seppo.nikkari@uta.fi

'Department of Medical Biochemistry, University of Tampere Medical School, Tampere, Finland

${ }^{3}$ Fimlab Laboratories, Tampere, Finland

Full list of author information is available at the end of the article
}

desaturase gene (FADS2, rs 3834458) that has been shown to lead to decreased plasma levels of arachidonic acid and eicosapentaenoic acid [2,3]. Polyunsaturated fatty acids (PUFA) may influence inflammation, as they are precursors to eicosanoids [4]. Especially the eicosanoids that are derived from arachidonic acid (20:4n-6), a metabolite of linoleic acid (18:2n-6), have mainly pro-inflammatory effects [4]. In addition to being precursors to eicosanoids, PUFA are readily auto-oxidized in the presence of oxygen. This process is accelerated by the presence of trace metals and resisted by chelating agents and antioxidants. Thus PUFA play roles both in regulation of inflammation, and as direct targets for peroxidation through their double bonds that promote oxidation. PUFA play an important role in atherosclerosis through regulation of cholesterol transport by lipoproteins, and oxidative modification of low-density lipoprotein (LDL) is a key event in the oxidation hypothesis of atherogenesis [5]. We have previously shown that HDL does not protect LDL from oxidation in vitro, but is in fact oxidized fastest of all lipoproteins
C Biomed Central 


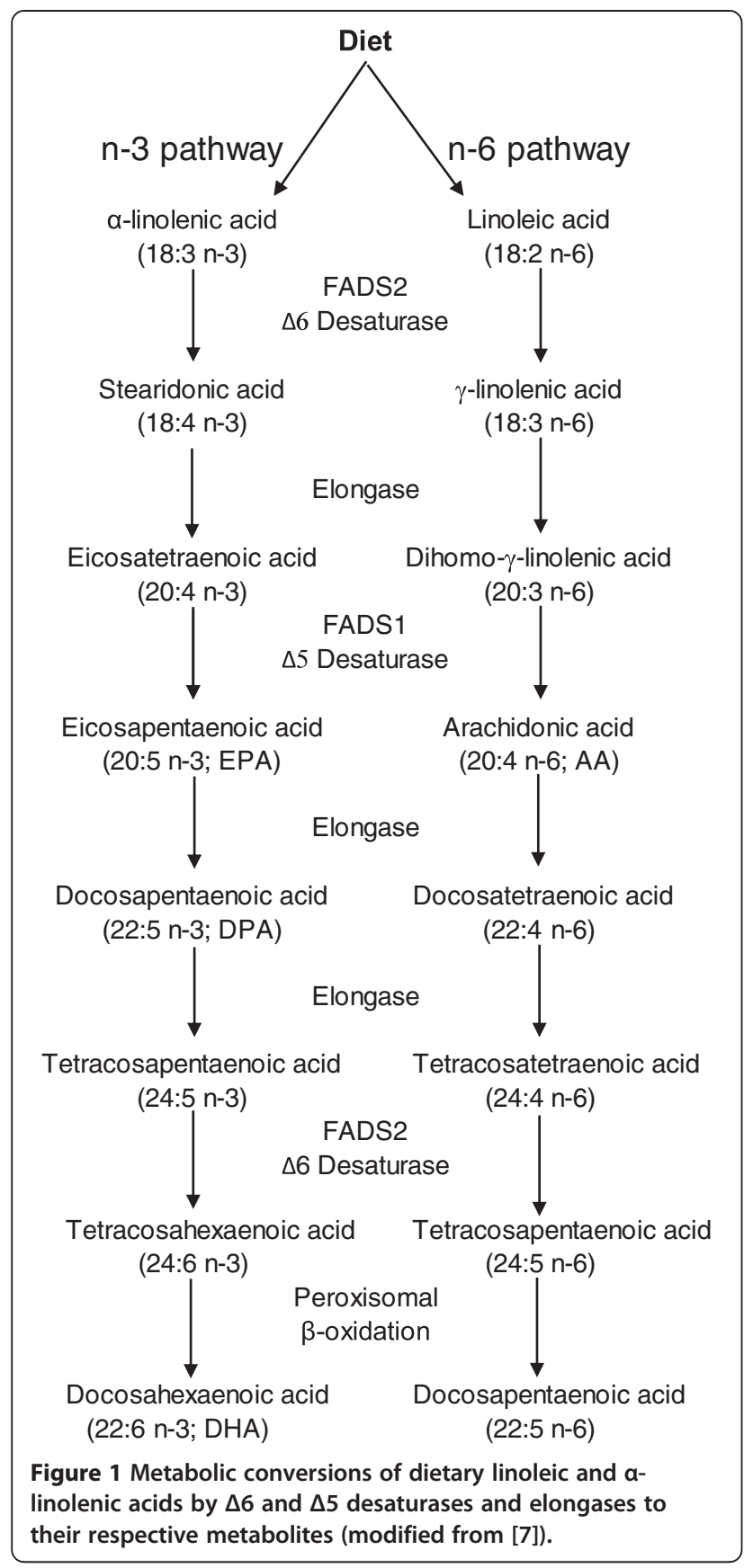

due to its rich polyunsaturated fatty acid (PUFA) composition, which is oxidation promoting [6]. In the present study, we tested the effect of FADS2 rs 3834458 on lipoprotein oxidation in vitro, in plasma derived from healthy subjects.

\section{Results}

Background characteristics of the subjects participating in the study are shown in Table 1 . The overall frequencies for the FADS2 insertion (T)/deletion (-) genotype groups in the study population were 0.24 for $\mathrm{T} / \mathrm{T}, 0.59$ for $\mathrm{T} /$,
Table 1 Characteristics of the study subjects $(n=58)$

\begin{tabular}{lc}
\hline Age (years) & $39.3 \pm 10.6$ \\
Body mass index $\left(\mathrm{kg} / \mathrm{m}^{2}\right)$ & $24.4 \pm 3.6$ \\
Total cholesterol $(\mathrm{mmol} / \mathrm{l})$ & $5.47 \pm 0.97$ \\
Triacylglycerol $(\mathrm{mmol} / \mathrm{l})$ & $1.35 \pm 0.71$ \\
$\mathrm{HDL}$ cholesterol $(\mathrm{mmo} / \mathrm{l})$ & $1.65 \pm 0.38$ \\
$\mathrm{LDL}$ cholesterol $(\mathrm{mmol} / \mathrm{l})$ & $3.21 \pm 0.96$
\end{tabular}

and 0.17 for $-/-$. The genotype distribution followed Hardy-Weinberg equilibrium.

Compared with subjects with [T/T] or [T/-] in FADS2 rs 3834458 , the peroxidizability index (Figure 2), the proportions of plasma total arachidonic acid (20:4n-6) (Figure 3) and eicosapentaenoic acid (20:5 n-3) (Figure 4) were significantly decreased in the subjects homozygous for the deletion [-/-]. Also docosapentaenoic acid (22:5 n-3) was similarly decreased $(\mathrm{p}=0.016)$ but not docosahexaenoic acid $(22: 6 n-3)(p=N S)$. The decreases for peroxidizability index, arachidonic acid, eicosapentaenoic acid and docosapentaenoic acid remained significant after adjusting for age and gender $(\mathrm{p}<0.001, \mathrm{p}<0.001, \mathrm{p}=0.008$ and $\mathrm{p}=0.010$ respectively). Plasma levels of linoleic acid and $\alpha$-linolenic acid did not correlate with the FADS2 rs 3834458 polymorphism $(\mathrm{p}=\mathrm{NS})$.

Genotypes [T/T] and [T/-] were combined for statistical analyses of the oxidation study since they showed similar

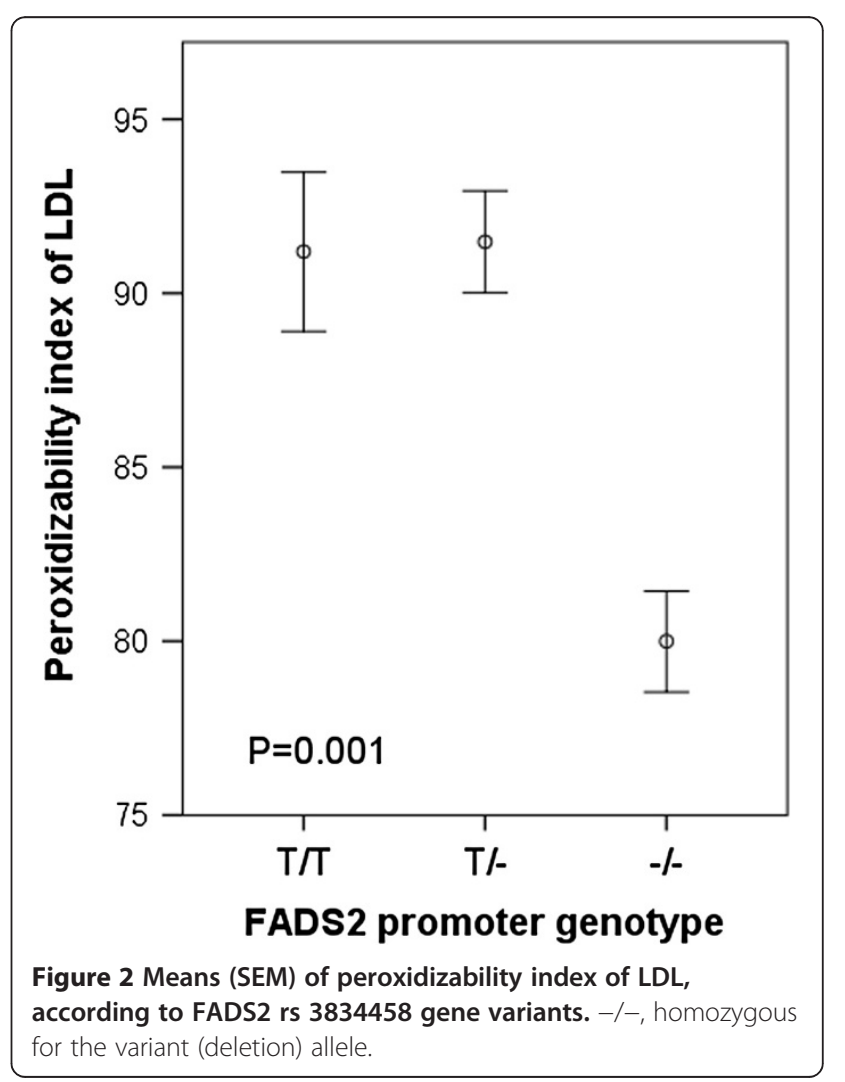




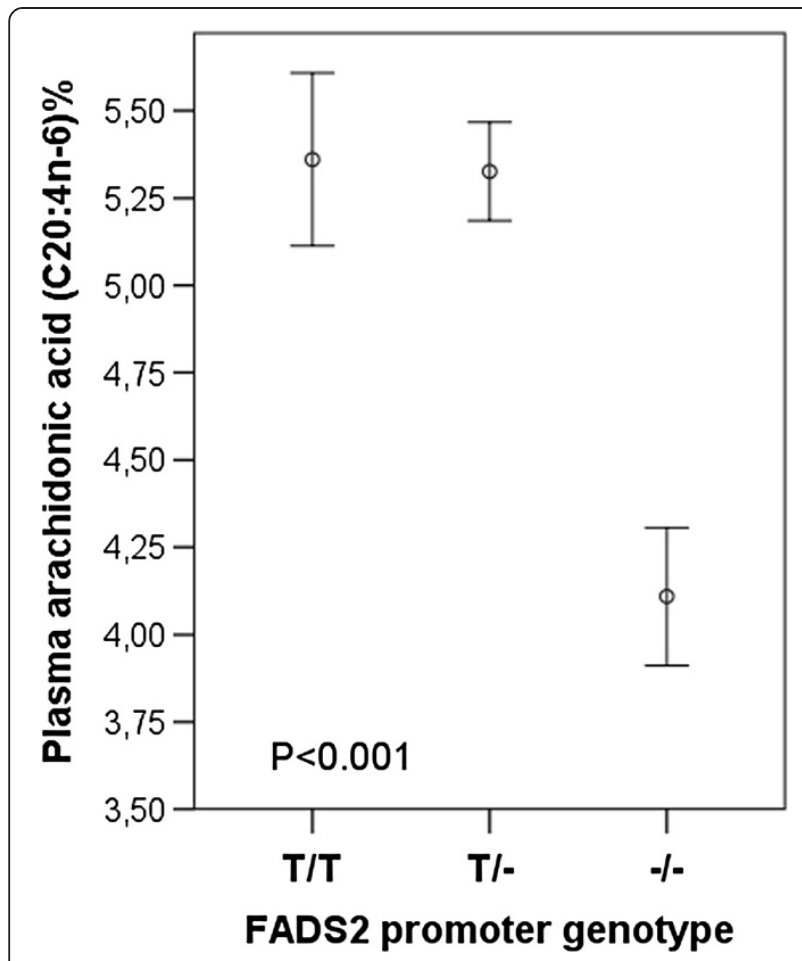

Figure 3 Means (SEM) of plasma arachidonic acid proportions, according to FADS2 rs 3834458 gene variants. $-/-$, homozygous for the variant (deletion) allele.

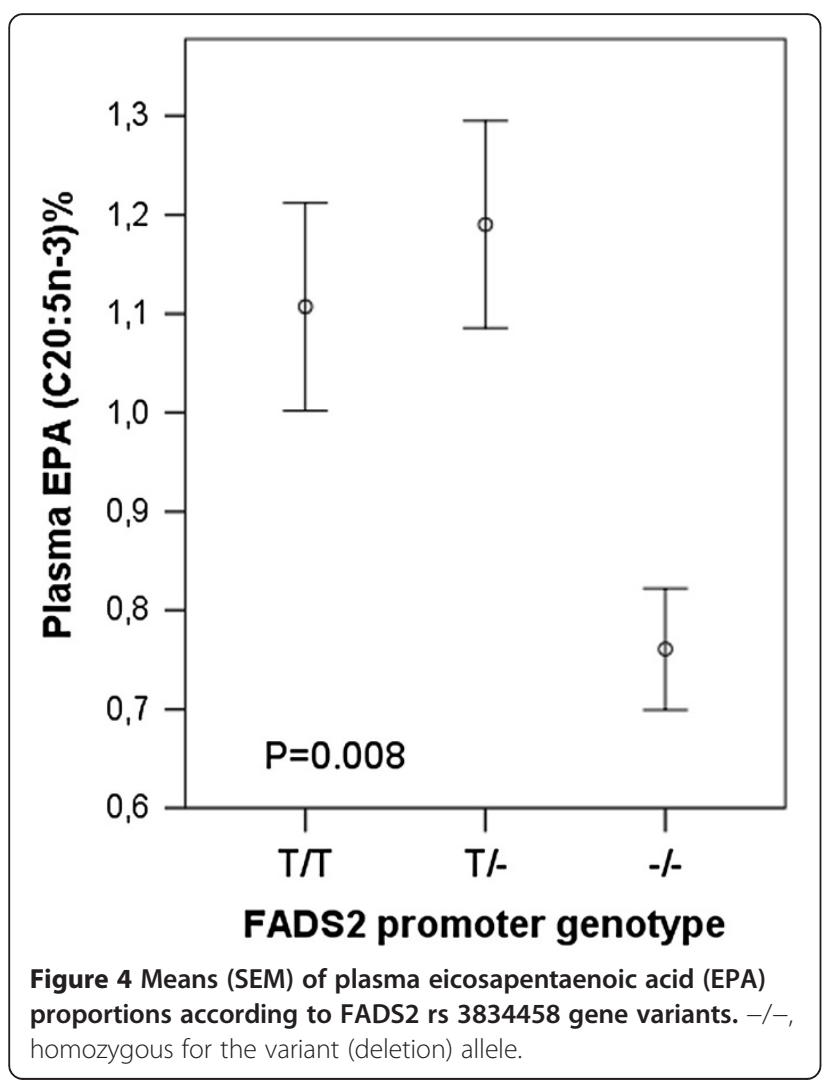

profiles for their effects on PUFA proportions. When mixtures of isolated $\mathrm{LDL}$ and $\mathrm{HDL}_{2}$ were subjected to $\mathrm{Cu}^{2+}$-induced oxidation, the subjects with [-/-] showed decreased rate of appearance $(\mathrm{p}=0.027)$ and the final concentration $(\mathrm{P}=0.033)$ of conjugated dienes, compared with $[\mathrm{T} / \mathrm{T}]$ and [T/-] (Table 2). The findings remained significant after adjusting for age and gender. When isolated $\mathrm{HDL}_{3}$ was subjected to $\mathrm{Cu}^{2+}$-induced oxidation with LDL, a similar trend was observed in oxidation rate, but only the final concentration of conjugated dienes was significantly decreased in subjects with $[-/-]$ compared with $[\mathrm{T} / \mathrm{T}]$ and $[\mathrm{T} / \mathrm{-}](\mathrm{p}=0.034 ; \mathrm{p}=0.031$ after adjusting for age and gender) (data not shown).

\section{Discussion}

The overall frequencies for the FADS2 rs 3834458 insertion $(\mathrm{T}) /$ deletion $(-)$ genotype groups in the study population were 0.24 for $\mathrm{T} / \mathrm{T}, 0.59$ for $\mathrm{T} / \mathrm{-}$, and 0.17 for $-/-$. These differed from the KOALA study (the Netherlands) and the LISA study (Germany), where the frequencies were $0.46,0.43,0.11$ and $0.47,0.44,0.09$, respectively [8]. In both of these studies, the FADS2 gene rs3834458 was typed with MALDI-TOF-MS. On the other hand, in subjects from Costa Rica, by using a variation of the allelespecific assay, the corresponding frequencies were 0.29 , 0.47 and 0.23 [3], which are more in line with our results. The difference in frequency in our study compared to the KOALA and LISA studies might be due to a different method of analysis, or to a difference in the study populations [8]. However, prior to analysis, we confirmed the products of our PCR primers to be specific for FADS2 rs 3834458 insertion comparing the sequenced products with the GenBank database.

Long-chain $n-3$ and $n-6$ polyunsaturated fatty acids are formed from the EFA linoleic acid and alpha-linolenic acid by sequential desaturation and elongation (Figure 1). There is a decreased promoter activity in FADS2 rs3834458 for this function [2]. As expected, the fatty acid profile of the plasma from subjects homozygous for the FADS2 rs 3834458 deletion $[-/-]$ differed substantially from that of

Table 2 Kinetic parameters of $\mathrm{LDL}+\mathrm{HDL}_{2}$ oxidation in healthy subjects according to FADS2 genotype

\begin{tabular}{lcccc}
\hline Genotype & TT and T - & -- & $\mathbf{P}$ & $\mathbf{P *}^{*}$ \\
$\mathbf{N}$ & $\mathbf{4 8}$ & $\mathbf{1 0}$ & & \\
\hline LDL + HDL & & & & \\
Lag time $(\mathrm{min})$ & $56.1 \pm 6.2$ & $57 \pm 5.4$ & 0.764 & 0.296 \\
Rate $(\mu \mathrm{mol} / / \mathrm{min})^{\mathrm{a}}$ & $0.685 \pm 0.075$ & $0.656 \pm 0.085$ & 0.027 & 0.027 \\
Max $(\mathrm{nmol} / \mathrm{mg})^{\mathrm{b}}$ & $777 \pm 50$ & $735 \pm 74$ & 0.033 & 0.034 \\
\hline
\end{tabular}

Values are mean $\pm \mathrm{SD} .{ }^{a}$ Rate means maximal formation rate of conjugated dienes during oxidation. Calculation of the diene concentration is based on $\varepsilon_{234 \mathrm{~nm}}=29500 \mathrm{I} / \mathrm{mol} / \mathrm{cm}$ of the conjugated dienes. ${ }^{\mathrm{b}}$ Max is the maximal amount of dienes produced per $\mathrm{mg}$ of LDL protein.

*; adjusted for age and gender. 
the other genotypes in that proportions of plasma total eicosapentaenoic acid (20:5 n-3), docosapentaenoic acid $(22: 5 n-3)$ and arachidonic acid (20:4 n-6) were significantly decreased. These findings are in line with earlier observations $[3,8,9]$. However, only about $8 \%$ of the dietary linoleic acid / $\alpha$-linolenic acid go through the elongase/desaturase biosynthetic pathway to eicosapentaenoic acid and conversion to docosahexaenoic acid is extremely low $(<0.1 \%)[10]$. This may put into perspective the clinical relevance of the genotype findings. Moreover, the type of fat in the diet is reflected in serum fatty acids [11] and we had no knowledge on the habitual diet of the participants, which could have influenced the outcome. In spite of this limitation, it seems unlikely that the subjects homozygous for the FADS2 rs 3834458 deletion [-/-] would have had a diet that differed substantially from that of the other genotypes.

Earlier studies have shown that there are several intrinsic properties of lipoproteins that can affect their susceptibility to oxidation. Especially in supplementation studies, lipoprotein antioxidant content and fatty acid composition [12-15] have been shown to have an impact on oxidation parameters. We analyzed the fatty acid compositions of $\mathrm{LDL}, \mathrm{HDL}_{2}$ and $\mathrm{HDL}_{2}$ particles and united the information in the fatty acid profiles into a single term - the peroxidizability index - which describes the combined reactivity of fatty acids towards reactive oxygen species [16]. The results of our experiments suggest that proportions of polyunsaturated fatty acids affected by FADS2 are related to the peroxidation index, oxidation rate and the amount of dienes formed during in vitro oxidation. This may also have implications in vivo since - although PUFA are considered to be beneficial in many aspects - they also have inflammatory and pro-oxidant properties.

\section{Conclusions}

In summary, we report that compared to subjects with [T/ $\mathrm{T}]$ or [T/-] in FADS2 rs 3834458, the formation of arachidonic acid and EPA were significantly decreased, as reported previously. What is new is that the theoretical peroxidizability index was also decreased in lipoproteins from these individuals. Consequently, when the isolated LDL and HDL were subjected to $\mathrm{Cu}^{2+}$-induced oxidation, the subjects with [-/-] showed decreased rate of appearance and the final concentration of conjugated dienes, compared to $[\mathrm{T} / \mathrm{T}]$ and $[\mathrm{T} /-]$. Thus, FADS2 genotype may play a role in peroxidation of lipoproteins.

\section{Methods}

\section{Subjects}

61 healthy subjects from the personnel and medical students of the Department of Medical Sciences of Tampere University and Tampere University Hospital volunteered. The age range of the subjects was 20 to 58 years. 33 were women and 28 were men. All participants filled in a questionnaire, where emphasis was given to their health status (diseases and use of medication) in addition to health related behaviour (smoking, use of alcohol and vitamins). The results of three of the participants were later removed from analysis because of reported bowel diseases. Thus, 58 subjects remained; 32 women and 26 men. All participants gave their written consent to the study. The study protocol was approved by the ethics committee of the Tampere University Hospital.

\section{Blood Samples}

Fasting (12 h) blood samples were taken into suitable tubes (Vacuette, Greiner) from the antecubital vein in a sitting position after a $15-$ min rest using minimal stasis. Samples for the analysis of lipids, extraction of DNA and isolation of lipoproteins were taken into pre-chilled EDTA tubes, which were immediately placed in ice. Plasma was separated after centrifugation (Heraeus, 2000xg, $+4^{\circ} \mathrm{C}$ ). EDTA plasmas for isolation of lipoproteins were supplemented with sucrose $(0.6 \% \mathrm{w} / \mathrm{v}$ final concentration). This procedure has been shown to preserve LDL from oxidation for at least two months and the oxidation curve does not differ from that of a fresh sample [17]. Lipids were analysed as described [6]. All samples were kept frozen at $-70^{\circ} \mathrm{C}$ until analyzed.

\section{DNA isolation, FADS2 genotyping and Sequencing}

Genomic DNA was extracted from peripheral blood leukocytes using a commercial kit according to the manufacturer's instructions (Qiagen, Hilden, Germany). Insertion/ deletion of $\mathrm{T}$ in FADS2 was genotyped with the use of 2allele specific primers designed in such a way that the only difference between them was the insertion site at the very last nucleotide of their 3 'ends. Prior to final genotyping the amplified products were confirmed by sequencing 8 clones from an individual who proved to be homozygous for insertion and 16 randomly selected clones from another one who was homozygous for deletion variant. After amplification, PCR products were run in $2 \%$ agarose gel electrophoresis. Two parallel PCRs were performed for each DNA sample. These contained primers: 5'CTAG GTGACGCCCTTCCTT 3' (right), and either 5'GAGG TTCCGCAATTCTTTTCT 3' (left) or 5'GAGGTTCC GCAATTCTTTTC 3' (left). Amplification conditions were; $94^{\circ} \mathrm{C} 15 \mathrm{~min}$, followed by 32 cycles of $94^{\circ} \mathrm{C}, 63^{\circ} \mathrm{C}$ and $72^{\circ} \mathrm{C}$ for $30 \mathrm{~s}$ each and final extension at $72^{\circ} \mathrm{C}$ for $5 \mathrm{~min}$. For control sequencing, PCR was performed using primers D6Dsense (5'-GCC AGT TCC TCA TCG CCC CC-3') and D6Dantisense (5'-TCC CTT CCC CAT GCT GCC TG-3') [2]. PCR conditions consisted of $15 \mathrm{~min}$ at $94^{\circ} \mathrm{C}$, followed by 33 cycles at $94^{\circ} \mathrm{C} 1 \mathrm{~min}, 69^{\circ} \mathrm{C} 1 \mathrm{~min}$, $72^{\circ} \mathrm{C} 2 \mathrm{~min}$, and final extension $72^{\circ} \mathrm{C}$ for $30 \mathrm{~min}$. The primers produced fragments of $1059 \mathrm{bp}$. Prior to sequencing, amplified PCR products were cloned by using the 
TOPO TA (Invitrogen) cloning system. The automated ABI PRISM 3130 Genetic Analyzer (Applied Biosystems) and BigDye Terminator Cycle sequencing chemistry (Applied Biosystems) using M13(-20)F and M13R primers for determining DNA sequences on both strands. The partial $16 \mathrm{~S}$ rDNA sequences of approximately $600 \mathrm{bp}$ were edited and aligned using the Chromas 2.31 (Technelysium) and ClustalW sequence analysis software, and compared with those in the GenBank database by using the BLAST search tool (National Center for Biotechnology Information; http://www.ncbi.nlm.nih.gov/BLAST/).

\section{Isolation of lipoproteins and plasma fatty acid composition}

Lipoproteins were fractionated by isopycnic density gradient ultracentrifugation using a Beckman SW40 Ti rotor in a Beckman L60 centrifuge $(36000 \mathrm{rpm}, 40$ hours, $10^{\circ} \mathrm{C}$ ) as previously described [6]. The fatty acid compositions were analyzed by capillary gas-liquid chromatography [6,7]. From fatty acid compositions, the peroxidizability index was calculated: $(\mathrm{PI})=[(\Sigma \mathrm{mol} \%$ monoenoic FAs $\times 0.025)+(\Sigma$ mol\% dienoic FAs $\times 1)+$ $(\Sigma$ mol\% trienoic FAs $\times 2)+(\Sigma$ mol\% tetraenoic FAs $\times 4)+$ $(\Sigma$ mol\% pentaenoic $\mathrm{FAs} \times 6)+(\Sigma$ mol\% hexaenoic FAs $\times 8)][14]$.

\section{Oxidation of lipoproteins}

The susceptibility of mixtures of $\mathrm{LDL}$ and $\mathrm{HDL}_{2}$ or $\mathrm{HDL}_{3}$ subtractions to in vitro copper-catalyzed oxidation was assessed by continuously monitoring the production of conjugated dienes at $234 \mathrm{~nm}$, as previously described $[6,18]$. Oxidation was started by adding $10 \mu \mathrm{l}$ of $\mathrm{CuSO} 4$ to a final concentration of $1.65 \mu \mathrm{M} \mathrm{Cu}^{2+}$. The spectrophotometer was computer-operated (UVWinlab 2.1). This program also collected the absorbance data at 2min intervals during the oxidation. Several characteristic oxidation indices were obtained from the resulting absorbance versus time curves $[17,19]$.

\section{Data analysis}

Results are expressed as mean \pm SD unless otherwise stated. Statistical comparisons were made by Univariate General Linear Model, T-test and Mann-Whitney test using IBM SPSS software, version 20. Due to skewed distribution, plasma values of eicosapentaenoic acid and kinetic parameter of $\mathrm{LDL}+\mathrm{HDL}_{2}$ oxidation rate were used as their logarithms but reported as their original results. A p value $<0.05$ was taken to be statistically significant.

\section{Competing interests}

The authors report no competing interests.

\section{Authors' contributions}

TS, OJ and STN had substantial contributions to conception and design and interpretation of data and writing the manuscript. TK and TL had substantial contributions to conception and design. TS, JR and TK carried out the biochemical analyses. All authors read and approved the final manuscript.

\section{Acknowledgements}

We appreciate the expert technical assistance of Mirka Pietiläinen, Nina Peltonen, Ulla Saarijoki and Marita Koli. This study was supported by grants from competitive research funding of the Pirkanmaa Hospital District, Finnish Association of Clinical Biochemistry and the Finnish Cultural Foundation.

\section{Author details}

'Department of Medical Biochemistry, University of Tampere Medical School, Tampere, Finland. ${ }^{2}$ Institute of Medical Technology, University of Tampere, Tampere, Finland. ${ }^{3}$ Fimlab Laboratories, Tampere, Finland.

Received: 26 March 2013 Accepted: 27 May 2013

Published: 30 May 2013

\section{References}

1. Das UN: Essential fatty acids and their metabolites could function as endogenous HMG-CoA reductase and ACE enzyme inhibitors, antiarrhythmic, anti-hypertensive, anti-atherosclerotic, anti-inflammatory, cytoprotective, and cardioprotective molecules. Lipids Health Dis 2008, 7:37.

2. Nwankwo JO, Spector AA, Domann FE: A nucleotide insertion in the transcriptional regulatory region of FADS2 gives rise to human fatty acid delta-6-desaturase deficiency. J Lipid Res 2003, 44:2311-2319.

3. Baylin A, Ruiz-Narvaez E, Kraft P, Campos H: Alpha-Linolenic acid, delta6desaturase gene polymorphism, and the risk of nonfatal myocardial infarction. Am J Clin Nutr 2007, 85:554-560.

4. Calder PC: Polyunsaturated fatty acids and inflammatory processes: New twists in an old tale. Biochimie 2009, 91:791-795.

5. Steinberg $D$, Witztum JL: Is the oxidative modification hypothesis relevant to human atherosclerosis? Do the antioxidant trials conducted to date refute the hypothesis? Circulation 2002, 105:2107-2111.

6. Solakivi T, Jaakkola O, Salomäki A, Peltonen N, Metso S, Lehtimäki T, Jokela H, Nikkari ST: HDL enhances oxidation of LDL in vitro in both men and women. Lipids Health Dis 2005, 4:25.

7. Solakivi T, Kaukinen K, Kunnas T, Lehtimäki T, Mäki M, Nikkari ST: Serum fatty acid profile in celiac disease patients before and after a gluten-free diet. Scand J Gastroenterol 2009, 44:826-830.

8. Rzehak P, Thijs C, Standl M, Mommers M, Glaser C, Jansen E, Klopp N, Koppelman GH, Singmann P, Postma DS, Sausenthaler S, Dagnelie PC, van den Brandt PA, Koletzko B, Heinrich J, for the KOALA and the LISA study group: Variants of the FADS1 FADS2 Gene Cluster, Blood Levels of Polyunsaturated Fatty Acids and Eczema in Children within the First 2 Years of Life. PLoS One 2010, 5:e13261.

9. Schaeffer L, Gohlke H, Müller M, Heid IM, Palmer L, Kompauer I, Demmelmair H, Illig T, Koletzko B, Heinrich J: Common genetic variants of the FADS1 FADS2 gene cluster and their reconstructed haplotypes are associated with the fatty acid composition in phospholipids. Hum Mol Genet 2006, 15:1745-1756.

10. Williams CM, Burdge G: Long-chain n-3 PUFA: plant v. marine sources. Proc Nutr Soc 2006, 65:42-50.

11. Nikkari T, Luukkainen P, Pietinen P, Puska P: Fatty acid composition of serum lipid fractions in relation to gender and quality of dietary fat. Ann Med 1995, 27:491-498.

12. Kunitake ST, Jarvis MR, Hamilton RL, Kane JP: Binding of transition metals by apolipoprotein A-I-containing plasma lipoproteins: inhibition of oxidation of low density lipoproteins. Proc Natl Acad Sci USA 1992, 89:6993-6997.

13. Kontush A, Chantepie S, Chapman MJ: Small, dense HDL particles exert potent protection of atherogenic LDL against oxidative stress. Arterioscler Thromb Vasc Biol 2003, 23:1881-1888.

14. Ziouzenkova O, Gieseg SP, Ramos P, Esterbauer H: Factors affecting resistance of low density lipoproteins to oxidation. Lipids 1996, 31:S71-S76.

15. Gieseg SP, Esterbauer H: Low density lipoprotein is saturable by prooxidant copper. FEBS Lett 1994, 343:188-194.

16. Lambert AJ, Portero-Otin M, Pamplona R, Merry BJ: Effect of ageing and caloric restriction on specific markers of protein oxidative damage and membrane peroxidizability in rat liver mitochondria. Mech Ageing Dev 2004, 125:529-538 
17. Ramos P, Gieseg SP, Schuster B, Esterbauer H: Effect of temperature and phase transition on oxidation resistance of low density lipoprotein. J Lipid Res 1995, 36:2113-2128.

18. Esterbauer H, Striegl G, Puhl H, Rotheneder M: Continuous monitoring of in vitro oxidation of human low density lipoprotein. Free Rad Res Commun 1989, 6:67-75.

19. Leinonen JS, Rantalaiho V, Solakivi T, Koivula T, Wirta O, Pasternack A, Alho $H$, Lehtimäki T: Susceptibility of LDL to oxidation is not associated with the presence of coronary heart disease or renal dysfunction in NIDDM patients. Clin Chim Acta 1998, 275:163-174.

doi:10.1186/1476-511X-12-80

Cite this article as: Solakivi et al:: Delta-6-desaturase gene polymorphism is associated with lipoprotein oxidation in vitro. Lipids in Health and Disease 2013 12:80

\section{Submit your next manuscript to BioMed Central and take full advantage of:}

- Convenient online submission

- Thorough peer review

- No space constraints or color figure charges

- Immediate publication on acceptance

- Inclusion in PubMed, CAS, Scopus and Google Scholar

- Research which is freely available for redistribution 